Journal of Advanced Research in Fluid Mechanics and Thermal Sciences

\title{
Suction Effect on Stagnation Point Flow and Heat Transfer over an Exponentially Shrinking Sheet in a Porous Medium
}

\author{
Nirwana Japili ${ }^{1}$, Haliza Rosali ${ }^{1,}{ }^{*}$, Norfifah Bachok $^{1,2}$ \\ Department of Mathematics, Faculty of Science, Universiti Putra Malaysia, 43400 UPM Serdang, Selangor, Malaysia \\ Institute for Mathematical Research, Universiti Putra Malaysia, 43400 UPM Serdang, Selangor, Malaysia
}

ARTICLE INFO ABSTRACT

\section{Article history:}

Received 19 March 2020

Received in revised form 8 May 2020

Accepted 14 May 2020

Available online 31 July 2020

\section{Keywords:}

Exponentially stretching/shrinking; Heat transfer; Porous medium; Stagnationpoint flow, Suction/injection

\begin{abstract}
An investigation has been conducted to study the steady stagnation point flow and heat transfer in a porous medium caused by an exponentially stretching/shrinking sheet. Using a similarity transformation, the governing system of partial differential equations are transformed into a system of ordinary differential equations. The system of ordinary differential equations is then solved numerically using a shooting method in Maple software. The effect of the governing parameters on the skin friction coefficients and the local Nusselt numbers as well as the velocity and temperature profiles are analysed and discussed. Results obtain in this study are illustrated graphically. The range of parameter $C$ where the similarity solution exists for the steady stagnation point over an exponentially shrinking sheet in porous medium is larger than linear shrinking sheet.
\end{abstract}

Copyright @ 2020 PENERBIT AKADEMIA BARU - All rights reserved

\section{Introduction}

Over the last few decades, the study of the shrinking aspect has gained interest from various researchers to study the change in the behavior of fluid flow over a shrinking sheet. Wang [1] was the first researcher that initiates the discussion of the fluid flow towards a shrinking sheet. Later on, Miclavčič and Wang [2] have proven the existence of solutions in numerical and exact solutions for a few values of the suction parameter. Since then, the work of Wang has been extended to many other researchers by considering many different physical conditions. After that, Wong et al., [3] have proven the numerous unique characteristics of the shrinking sheet problem. In addition, Nadeem and Awais [4] were also interested in studying the flow over the shrinking sheet in a porous medium. At a later date, Ishak et al., [5] were concerned about the numerical solution for the micropolar fluid

\footnotetext{
* Corresponding author.

E-mail address: liza_r@upm.edu.my

https://doi.org/10.37934/arfmts.73.2.163174
} 
flow over the shrinking sheet and found that the solutions obtained were not unique, which differed from the stretching case.

Many of the shrinking problem that was studied considered only the linear or nonlinear shrinking sheet, while the study of exponential shrinking sheet is relatively limited although it is significantly important in many engineering applications. Wong et al., [3] have studied the stagnation point flow over an exponentially stretching/shrinking. In extension of that, Bhattacharyya [6] investigated the flow and heat transfer over an exponentially shrinking sheet where it is shown that if the value of suction parameter is greater than the critical value, there is a possibility that the fluid flow will be in a steady state. Later on, Bhattacharyya and Vajravelu [7] studied stagnation point flow and heat transfer over an exponentially shrinking sheet. Bachok et al., [8] further investigated the boundary layer stagnation point flow and heat transfer over an exponentially stretching/shrinking sheet in a nanofluid and it is found that the range of similarity solutions that exist in exponential shrinking case is larger than in the linear shrinking sheet. Rohni et al., [9] considered the flow to be due to exponentially vertical shrinking sheet with the effect of suction. In addition, Kasmuri et al., [10] conducted the study of the boundary layer stagnation point flow and heat transfer past a permeable exponentially shrinking sheet. While, Rosali et al., [11] considered a rotating flow over an exponentially shrinking sheet with a suction effect. Merkin et al., [12] explored the stagnation-point flow and heat transfer over an exponentially stretching/shrinking cylinder and reported that as the curvature parameter increases, the heat transfer rate also increases and the solution for a shrinking cylinder is found to be non-unique.

In recent years, Kamal et al., [13] have performed the stability analysis on the stagnation-point flow and heat transfer over a permeable stretching/shrinking sheet with heat source effect and it is noticed that additional energy is generated in the boundary layer in the presence of heat source. Rahman et al., [14] analysed the steady 2-dimensional stagnation-Point Flow over an Exponentially Stretching/Shrinking Sheet in nanofluid with the effects of magnetohydrodynamic (MHD). They found that the skin friction coefficient increases as the magnetic field parameter increases. Furthermore, Lund et al., $[15,16]$ investigated the magnetohydrodynamic fluid flow towards an exponentially stretching/shrinking sheet. In the same year, Lund et al., [17] investigated the DarcyForchheimer flow of Casson type nanofluid over an exponentially sheet. Afterwards, Wahid et al., [18] investigated the flow of magnetohydrodynamic Casson fluid over a linear stretching surface in porous medium with slip condition. Mohamad et al., [19] studied the effect of suction on the stagnation point flow and heat transfer over a stretching/shrinking sheet in a porous medium along with the stability analysis. They reported that dual solutions exist for a certain range of shrinking case and these results are in excellent agreement with previous studies. Wahid et al., [20] examined magnetohydrodynamic (MHD) slip Darcy flow of viscoelastic fluid over a stretching surface in a porous medium with the presence of thermal radiation and viscous dissipation. They found that the skin friction coefficient increases for larger magnetic field and porosity parameter. Lund et al., [21] recently have conducted a study to examine the effect of Joule heating and viscous dissipation on MHD flow of micropolar fluid over an exponentially shrinking sheet. From this study, it is found that two solutions exist for the case of Newtonian fluid whereas three solutions exist for the case of nonNewtonian fluid.

The problem of suction or injection has become significantly important in various sectors of industry. Suction or injection is found to be important in delaying the separation of boundary layers in order to reduce drag. For example, suction or injection through the fluid will change the flow field as in the mass transfer cooling where it will ultimately influence the rate of heat transfer of the plate. Generally, the skin friction coefficient and heat transfer will increase due to the effect of suction 
whereas the opposite is shown in for the case of injection. This suction or injection theory has played an important role in many engineering applications, for example in the thermal oil recovery process.

Motivated by all these investigations, the purpose of this present paper is to investigate the stagnation point flow and heat transfer over an exponentially shrinking sheet in a porous medium with suction effect. In order to understand the characteristics of fluid flow and heat transfer, results for the skin friction coefficient, the local Nusselt number, the velocity profiles as well as the temperature profiles are obtained and discussed for different values of governing parameters.

\section{Methodology}

We consider a steady, laminar and two-dimensional boundary layer stagnation-point flow of an incompressible viscous fluid over an exponentially shrinking sheet in a porous medium (see Figure 1). We assumed that the free stream and the stretching/shrinking velocities are respectively in the forms $U_{e}=A \exp \left(\frac{x}{L}\right)$ and $U_{w}=B \exp \left(\frac{x}{L}\right)$ where $A$ and $B$ are constants, $x$ is the coordinate measuring distance along the stretching/shrinking sheet and $L$ is a characteristics length scale (see Stewartson [22]). We note that $B>0$ and $B<0$ correspond to stretching and shrinking sheet. Under these assumptions, the governing equations of motion and the energy are written as

$\frac{\partial u}{\partial x}+\frac{\partial v}{\partial y}=0$

$u \frac{\partial u}{\partial x}+v \frac{\partial u}{\partial y}=U_{e} \frac{d U_{e}}{d x}+v \frac{\partial^{2} u}{\partial y^{2}}+\frac{v}{K_{1}}\left(U_{e}-u\right)$

$u \frac{\partial T}{\partial x}+v \frac{\partial T}{\partial y}=\alpha \frac{\partial^{2} T}{\partial y^{2}}$

where $u$ and $v$ are the velocity components in $x-$ and $y$ - directions respectively, $v(=\mu / \rho)$ is the kinematic viscosity, $\rho$ is the density, $\mu$ is the coefficient of viscosity, $T$ is the fluid temperature, $\alpha$ is the thermal diffusivity and $K_{1}$ is permeability of the porous medium. The boundary conditions are given by:

$$
\begin{aligned}
& u=U_{w}, v=V_{w}=V_{0} \exp \left(\frac{x}{2 L}\right) \\
& T=T_{w}=T_{\infty}+T_{0} \exp \left(\frac{x}{2 L}\right) \quad \text { at } y=0 \\
& u \rightarrow U_{e}(x), \quad T \rightarrow T_{\infty} \quad \text { as } y \rightarrow \infty
\end{aligned}
$$

where $T_{w}$ is the variable temperature at the sheet and $T_{\infty}$ is the free stream temperature assumed to be constant.

The nonlinear system of partial differential Eq. (1) to (3) subject to the boundary conditions in Eq. (4) can be expressed in a simpler form by introducing the given similarity transformation (see Bhattacharyya and Vajravelu [7] and Bachok et al., [8]): 


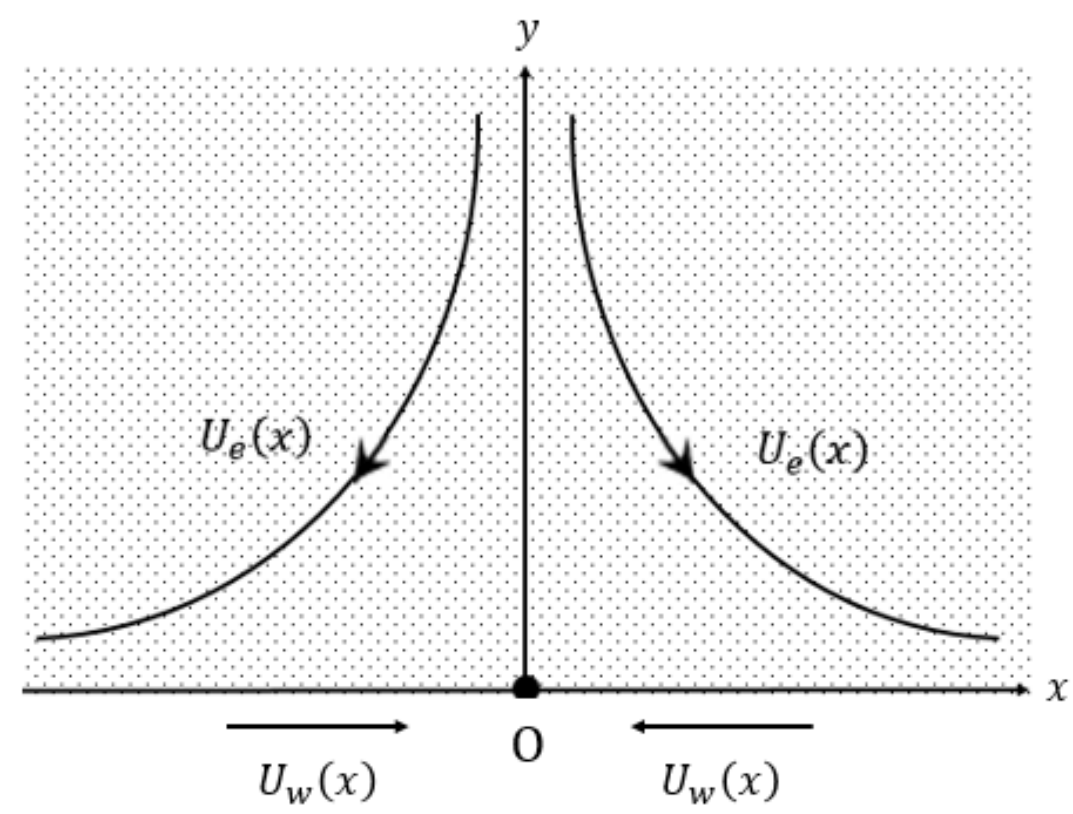

Fig. 1. Physical model of two-dimensional stagnation point flow towards an exponentially stretching/shrinking sheet

$\eta=y\left(\frac{A}{2 v L}\right)^{\frac{1}{2}} \exp \left(\frac{x}{2 L}\right)$

$\psi=(2 v L A)^{\frac{1}{2}} f(\eta) \exp \left(\frac{x}{2 L}\right)$

$\theta(\eta)=\frac{T-T_{\infty}}{T_{w}-T_{\infty}}$

where $\eta$ is the similarity variable and $\psi$ is the stream function defined as $u=\frac{\partial \psi}{\partial y}$ and $v=-\frac{\partial \psi}{\partial x^{\prime}}$, which identically satisfies Eq. (1). Substituting Eq. (5) into Eq. (2) to (4), we get the following system of differential equations

$f^{\prime \prime \prime}+f f^{\prime \prime}+2-2\left(f^{\prime}\right)^{2}+2 K\left(1-f^{\prime}\right)=0$

$\frac{1}{P r} \theta^{\prime \prime}+f \theta^{\prime}-f^{\prime} \theta=0$

subject to the boundary conditions

$$
\begin{aligned}
& f(0)=S, \quad f^{\prime}(0)=\frac{B}{A}=C, \quad \theta(0)=1 \\
& f^{\prime}(\eta) \rightarrow 1, \quad \theta(\eta) \rightarrow 0 \quad \text { as } \quad \eta \rightarrow \infty
\end{aligned}
$$

where prime denotes differentiation with respect to $\eta$. Here $\operatorname{Pr}=v / \alpha$ is the Prandtl number and $K=v /\left(A K_{1}\right)$ is the permeability parameter. The mass transfer parameter is given by $S=$ $-V_{0} \sqrt{2 L / v A}$ where $S>0$ is for suction and $S<0$ is for injection. Parameter $C$ is referring to stretching when $C>0$ and shrinking when $C<0$ and is defined as $C=B / A$. The skin friction coefficients and the local Nusselt number can be expressed as 


$$
C_{f}=\frac{\tau_{w}}{\rho U_{e}} \quad, \quad N u_{x}=\frac{L q_{w}}{k\left(T_{w}-T_{\infty}\right)}
$$

where the shear stress $\tau_{w}$ and the surface heat flux $q_{w}$ are given by

$$
\tau_{w}=\mu\left(\frac{\partial u}{\partial y}\right)_{y=0}, \quad q_{w}=-k\left(\frac{\partial T}{\partial y}\right)_{y=0}
$$

with $\mu$ and $k$ are the coefficient of viscosity and thermal conductivity, respectively. Using the similarity variables given by Eq. (5), we obtain

$2 C_{f} R e_{x^{\frac{1}{2}}}=f^{\prime \prime}(0)$

$\sqrt{2} N u_{x}=-\theta^{\prime}(0) R e_{x}^{-\frac{1}{2}}$

where $R e_{x}=\frac{U_{e} L}{v}$ is the local Reynolds number.

\section{Results and Discussion}

The nonlinear ordinary differential Eq. (6) and (7) satisfying the boundary conditions in Eq. (4) were solved numerically for some values of governing parameters by using shooting techniques in MAPLE software. To check the validity of the findings, we have compared our results with those reported by Wang [1] and Ishak et al., [5] when $\operatorname{Pr}=1, K=0$ and $S=0$ which means there is no suction effect is taken into account for different values of stretching/shrinking parameter $C$. For different values of $C$, the present results agree to six decimal places and that is consistent with the previously reported results by Wang [1] and Ishak et al., [5]. The comparison showed an excellent agreement as presented in Table 1.

The variations of $f^{\prime \prime}(0)$ and $-\theta^{\prime}(0)$ with $C$ are shown in Figure 2 to 5 for some values of $S$. In this particular study, there are three values of $S$ that have been considered for each cases which are $S=$ $0.3,0.5$ and 0.8 for the case of suction and $S=-0.3,-0.5$ and -0.8 for the case of injection. Figure 2 and 3 show the effect of mass transfer parameter $S$ on the skin friction coefficient $f^{\prime \prime}(0)$ and the local Nusselt number $-\theta^{\prime}(0)$ respectively, with parameter $C$ when $K=0.5, \operatorname{Pr}=1$ and $S=0.3,0.5$ and 0.8 . From these figures, it is observed that skin friction coefficient and the local Nusselt number increases with the increase in parameter $S$. Physically, this is due to the suction effect which increases the shear stress on the surface and therefore increases the velocity gradient at the surface which is compliance with Figure 2. This observation is in agreement with the fact that the fluids tend to move faster in the presence of the suction effect. In addition, increase in parameter $S$ will lead to an increase in the temperature gradient at the surface which indicates the heat transfer rate at the surface. However, opposite effect illustrated in Figure 4 and 5 where the values of skin friction coefficient and local Nusselt number found to be decreasing with the increase of injection parameter $S$ where $S=-0.3,-0.5$ and -0.8 .

It is also shown that for both suction and injection, dual solutions exist for a certain range of values $\left(C_{c}<C<0\right)$ for shrinking case, whereas the solution is found to be unique for stretching case $(C>0)$. Table 2 presents the critical values of $C$ where $C_{c}<0$ for which there will be no solutions to Eq. (6) and (7) subjected to Eq. (8) for $C<C_{c}$. From the table, it is also can be seen that as the parameter $S$ increases, the range of value in which the solution exists for Eq. (6) and (7) also increases. It is obviously shows that the effect of suction is to delay the boundary layer separation. 
The values of $f^{\prime \prime}(0)$ and $-\theta^{\prime}(0)$ for the present problem are tabulated in Table 3 and 4 for future reference.

Table 1

Comparison of the values of $f^{\prime \prime}(0)$ for various values of $C$ when $\operatorname{Pr}=1$

\begin{tabular}{lllll}
\hline \multicolumn{1}{c}{$C$} & Wang $[1]$ & Ishak et al., [5] & Present Results & $K=1.3$ \\
\hline-1.2465 & & & $K=0$ & \multicolumn{1}{c}{$K=0775$} \\
-1.15 & 0.5543 & 0.584295 & 0.584357 & 2.770775 \\
-1 & 1.08223 & 1.082231 & 1.082244 & 2.738766 \\
-0.75 & 1.32882 & 1.328817 & 1.328819 & 2.668088 \\
-0.5 & $1.4893-0$ & 1.489298 & 1.489299 & 2.499344 \\
-0.25 & 1.495670 & 1.495670 & 1.495670 & 2.274017 \\
0 & 1.40224 & 1.402241 & 1.402241 & 1.998308 \\
0.1 & 1.232588 & 1.232588 & 1.232588 & 1.676941 \\
0.2 & 1.146561 & 1.146561 & 1.146561 & 1.536472 \\
0.5 & 1.05113 & 1.051130 & 1.051130 & 1.389506 \\
2 & 0.71330 & 0.713295 & 0.713294 & 0.911451 \\
5 & -1.8873 & -1.887307 & -1.887307 & -2.201200 \\
\hline
\end{tabular}

\section{Table 2}

Values of $C c$ for various values of $S$ when $\operatorname{Pr}=1$ and $K=0.5$

\begin{tabular}{ll}
\hline \multicolumn{1}{c}{$S$} & $C c$ \\
\hline-0.8 & -1.717 \\
-0.5 & -1.814 \\
-0.3 & -1.891 \\
0.3 & -2.176 \\
0.5 & -2.292 \\
0.8 & -2.484 \\
\hline
\end{tabular}

Table 3

Values of $f^{\prime \prime}(0)$ for different values of $C$ and $S$, for stretching $(C>0)$ and shrinking $(C<0)$ when $\operatorname{Pr}=1$ and $K=0.5$

\begin{tabular}{llll}
\hline$f^{\prime \prime}(0)$ & & & \multicolumn{1}{c}{$S=0.8$} \\
\hline-2.17 & $S=0.3$ & $S=0.5$ & 3.688296 \\
& 1.463573 & 2.601829 & $(-0.386231)$ \\
-2.14 & $(0.935886)$ & $(0.170142)$ & 3.754543 \\
& 1.841237 & 2.728319 & $(-0.458505)$ \\
-2 & $(0.568230)$ & $(0.047535)$ & 3.991763 \\
& 2.549706 & 3.146500 & $(-0.715140)$ \\
-1.8 & $(-0.083697)$ & $(-0.347189)$ & 4.182055 \\
& 3.018658 & 3.482118 & $(-0.898571)$ \\
-1.4 & $(-0.599927)$ & $(-0.608294)$ & 4.225607 \\
& 3.359343 & 3.698039 & $(-0.369709)$ \\
-1 & & & 3.965345 \\
-0.5 & 3.295335 & 3.556124 & 3.333159 \\
0 & 2.858954 & 3.043342 & 2.433737 \\
0.2 & 2.129271 & 2.247566 & 2.009582 \\
0.4 & 1.769246 & 1.862759 & 1.552644 \\
0.6 & 1.374345 & 1.443741 & 1.064385 \\
0.8 & 0.946674 & 0.992504 & 0.546377 \\
\hline
\end{tabular}

() second solution 


\section{Table 4}

Values of $-\theta^{\prime}(0)$ for different values of $C$ and $S$, for stretching $(C>0)$ and shrinking $\frac{(C<0) \text { when } P r=1 \text { and } K=0.5}{-\theta^{\prime}(0)}$

\begin{tabular}{llll}
$C$ & $S=0.3$ & $S=0.5$ & \multicolumn{1}{c}{$S=0.8$} \\
\hline-2.17 & -0.975855 & -0.387015 & 0.090972 \\
& $(-0.975855)$ & $(-2.390869)$ & $(-4.273465)$ \\
-2.14 & -0.742692 & -0.316044 & 0.131897 \\
& $(-1.699225)$ & $(-2.634757)$ & $(-4.712433)$ \\
-2 & -0.332351 & -0.069062 & 0.297002 \\
& $(-2.793040)$ & $(-3.808866)$ & $(-8.166168)$ \\
-1.8 & -0.031280 & 0.173463 & 0.485283 \\
& $(-4.876110)$ & $(-9.245486)$ & $(-95.893382)$ \\
-1.4 & 0.343758 & 0.509865 & 0.774048 \\
& & & $(2.816540)$ \\
-1 & 0.607561 & 0.758160 & 0.999238 \\
-0.5 & 0.866573 & 1.007023 & 1.231873 \\
0 & 1.080853 & 1.215302 & 1.430024 \\
0.2 & 1.158000 & 1.290670 & 1.502308 \\
0.4 & 1.231274 & 1.362408 & 1.571350 \\
0.6 & 1.301167 & 1.430957 & 1.637520 \\
0.8 & 1.368074 & 1.496678 & 1.701123 \\
\hline
\end{tabular}

() second solution

As illustrated in Figure 2 to 5 , it is shown that the range of $C$ in which the similarity solution exists is larger in the case of exponentially shrinking sheet as compared to the range of values $C$ where the solution exists for the linear shrinking case (see Bhattacharya and Vajravelu [7]). Hence, it is clear that in the exponential case, the shrinking rate is seems to be faster than the linear case. By referring to the Figures 2 and 4, it is also observed that for exponential case, the range of which dual solutions occur increases and the second solution found to be exists even when the rate of shrinking is as low as $(C=-1.5)$.

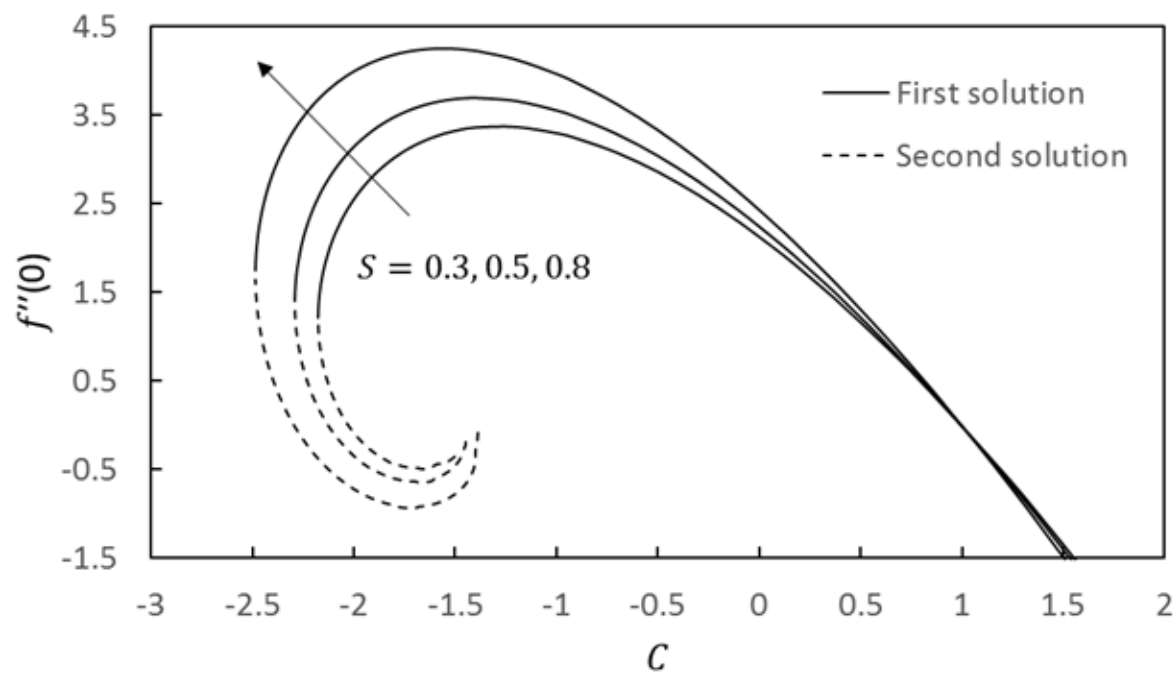

Fig. 2. The variation of the skin friction coefficient $f^{\prime \prime}(0)$ with $C$ for $S=$ $0.3,0.5$ and 0.8 when $\operatorname{Pr}=1$ and $K=0.5$ 


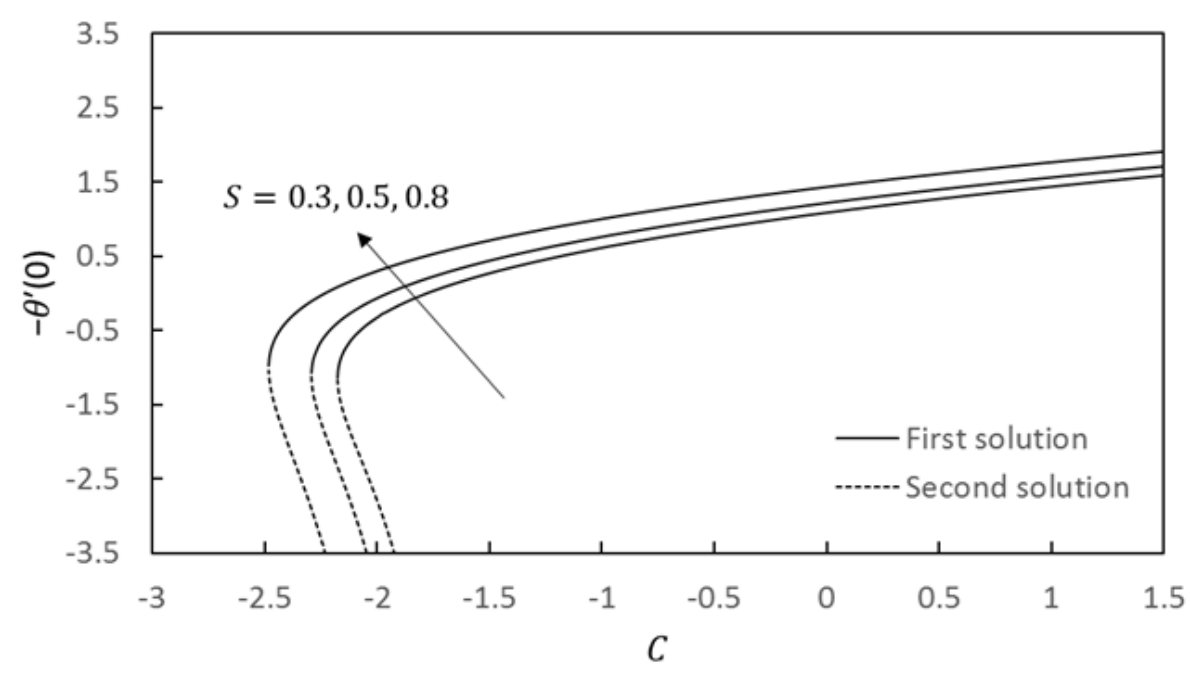

Fig. 3. The variation of the local Nusselt number $-\theta^{\prime}(0)$ with $C$ for $S=$ $0.3,0.5$ and 0.8 when $\operatorname{Pr}=1$ and $K=0.5$

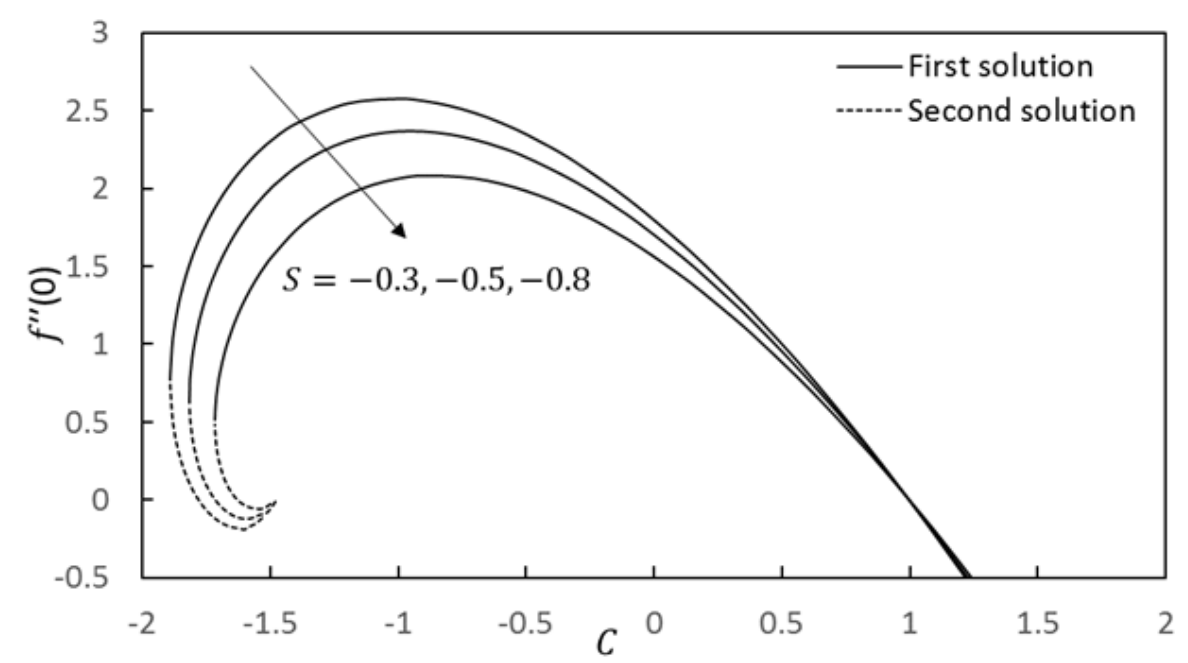

Fig. 4. The variation of the skin friction coefficient $f^{\prime \prime}(0)$ with $C$ for $S=$ $-0.3,-0.5$ and -0.8 when $\operatorname{Pr}=1$ and $K=0.5$

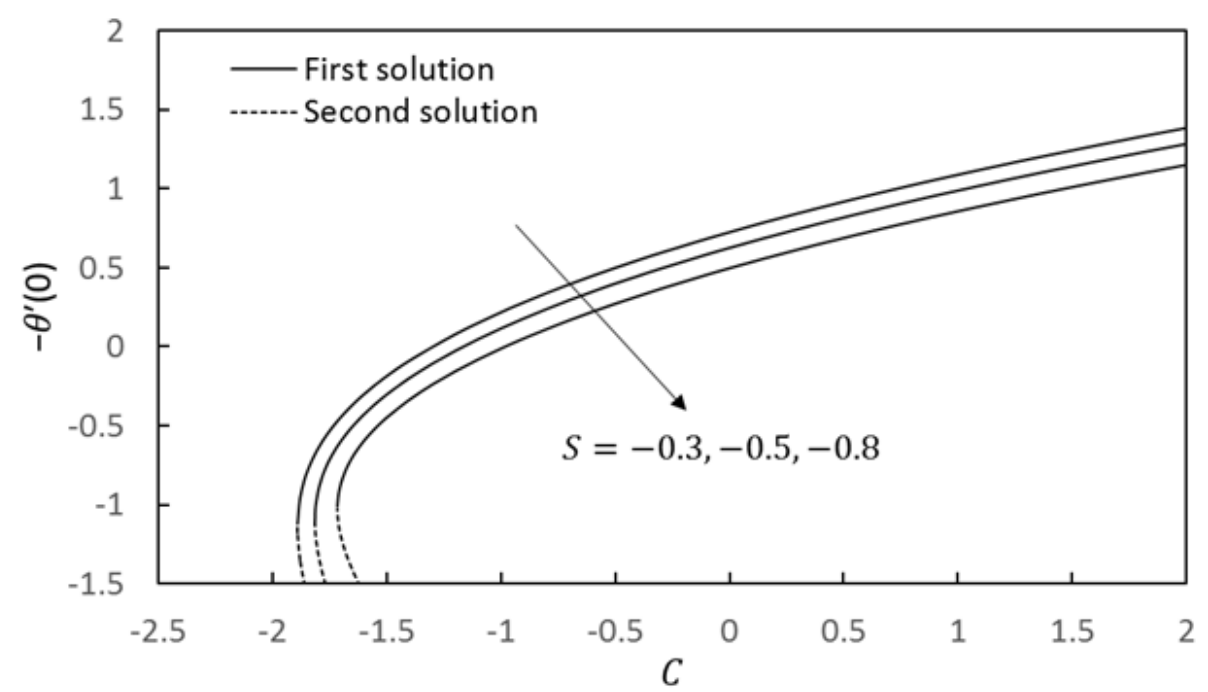

Fig. 5. The variation of the local Nusselt number $-\theta^{\prime}(0)$ with $C$ for $S=$ $-0.3,-0.5$ and -0.8 when $\operatorname{Pr}=1$ and $K=0.5$ 
Figure 6 and 7 show the velocity and temperature profiles respectively for the case of suction $(S>0$ ) when $C=-2.15$ (shrinking). From Figure 6 , it can be seen that as the value of suction parameter $S$ increases, the velocity profile also increases in the first solution but decreases as for the second solution. It is also found that the suction effect raises the thickness of the boundary layer as the parameter of $S$ increases. As a result, the surface shear stress increases hence, the skin friction increases as demonstrates in Figure 2. Figure 7 shows that the increase in the value of suction parameter $S$ will reduce the temperature profile as seen in the first solution but increases in the second solution. It is noticed that the suction effect reduces the thickness of the thermal boundary layer and in turn leads to an increase in the temperature gradient at the surface.

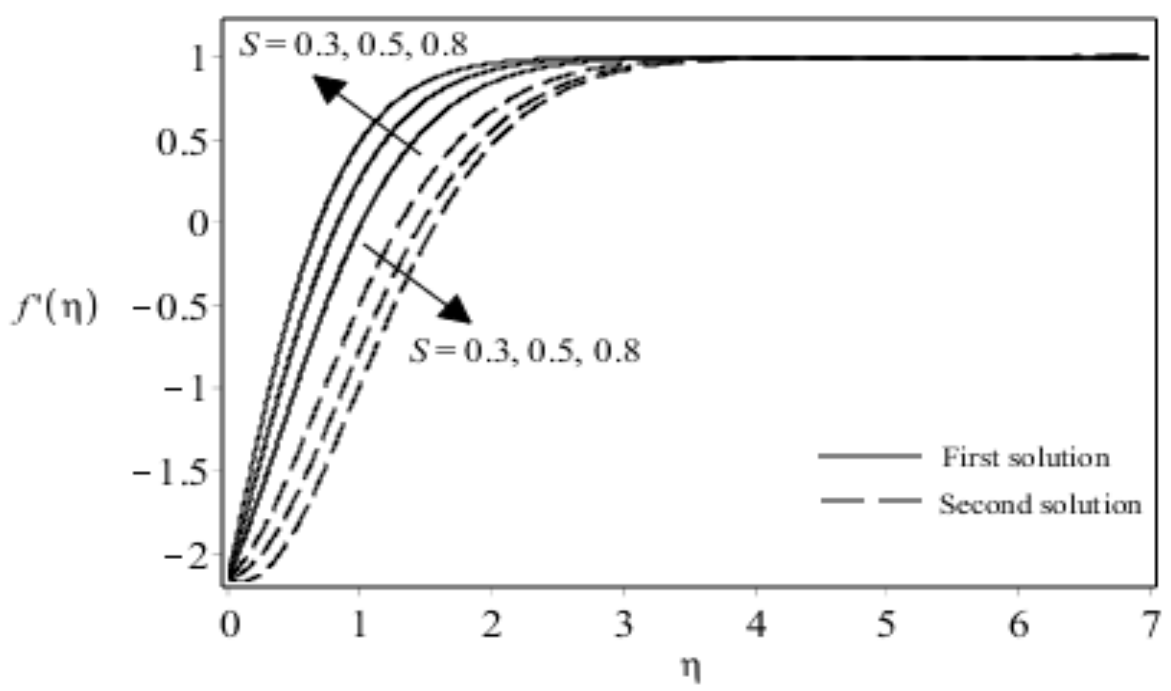

Fig. 6. Velocity profiles $f^{\prime}(\eta)$ for different values of $S$ when $C=-2.15$ (shrinking), $\operatorname{Pr}=1$ and $K=0.5$

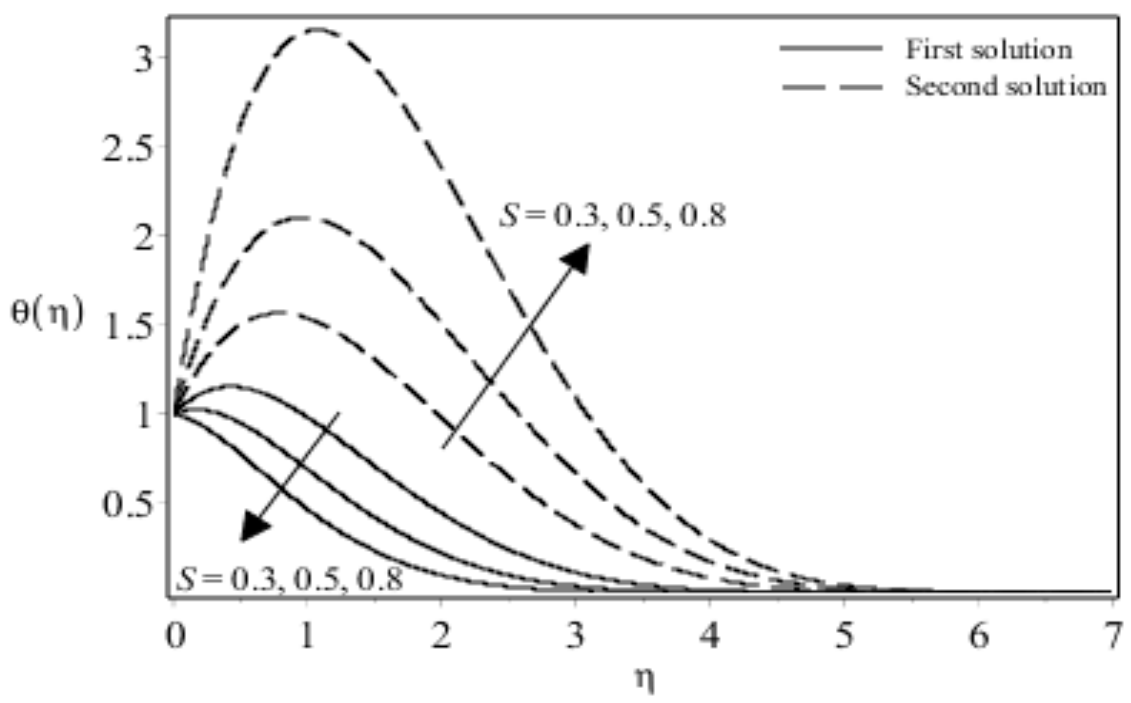

Fig. 7. Temperature profiles $\theta(\eta)$ for different values of $S$ when $C=$ -2.15 (shrinking), $\operatorname{Pr}=1$ and $K=0.5$

Whereas, Figure 8 and 9 present the velocity and temperature profiles respectively for the case of injection $(S<0)$ for certain values of $C<0$ (shrinking). From Figure 8 , it is shown that the velocity profiles decrease as the injection parameter $S$ increases for both first and second solutions. In Figure 9 , it shows that there is an increment in the temperature profile when the value of injection 
parameter $S$ increases for both first and second solutions. Therefore, in these figures, it can be observed that both of the first and second solution profiles asymptotically satisfied the far field boundary conditions. Hence, this confirmed the validity of the results obtained while supporting the dual nature of solutions shown in Figure 2 to 5.

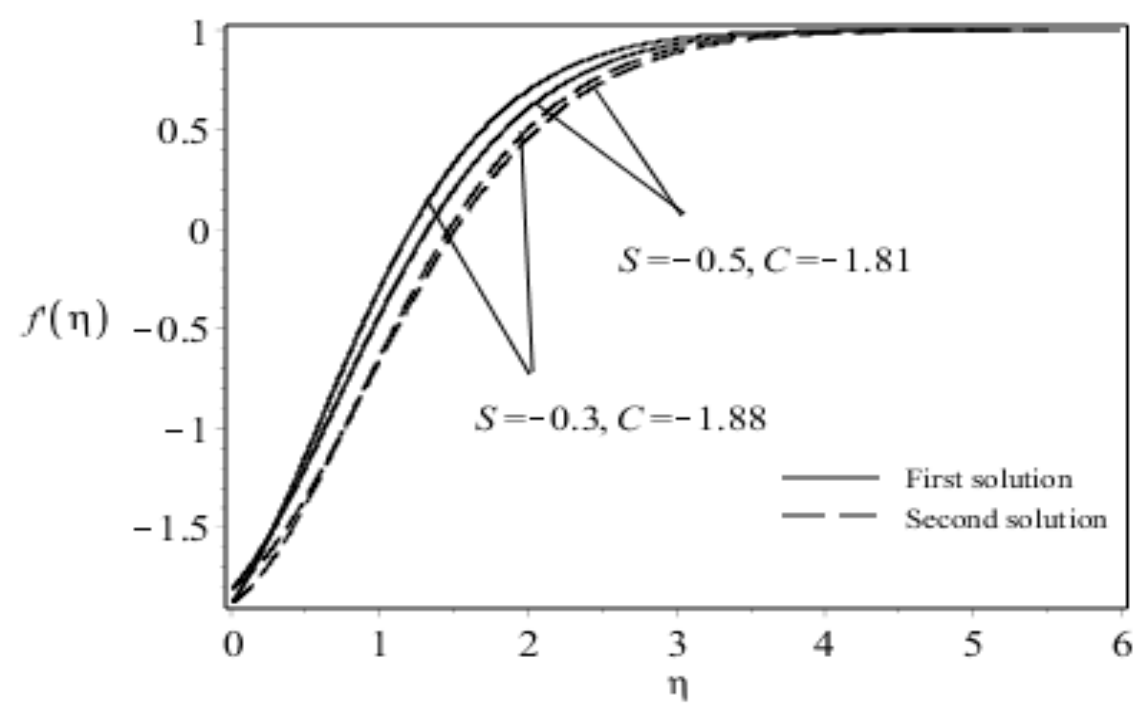

Fig. 8. Velocity profiles $f^{\prime}(\eta)$ for different values of $S$ and $C$ when $\operatorname{Pr}=$ 1 and $K=0.5$

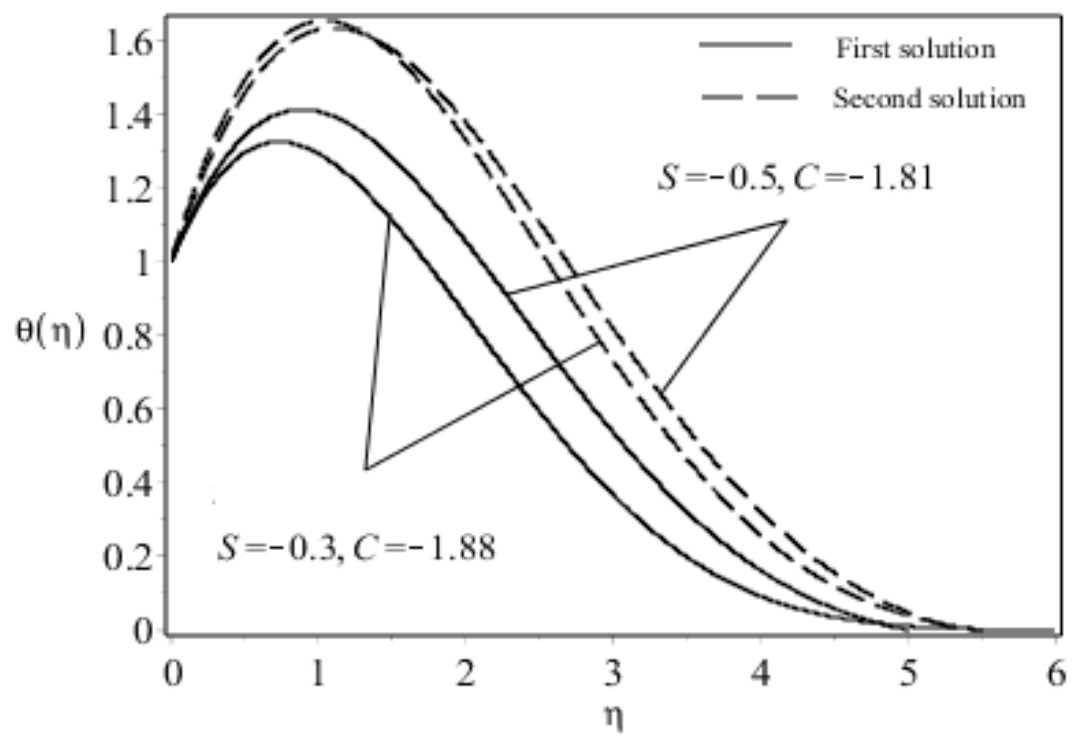

Fig. 9. Temperature profiles $\theta(\eta)$ for different values of $S$ and $C$ when $\operatorname{Pr}=1$ and $K=0.5$

\section{Conclusions}

In this paper, a numerical study has been performed for the case of steady stagnation point flow and heat transfer over an exponentially shrinking sheet in a porous medium with the effect of suction. The governing boundary layer equations were solved numerically using the shooting method. The skin friction coefficient and the local Nusselt number with the effect of suction have been obtained and analysed along with the velocity and the temperature profiles. The numerical solutions to the 
present problem are found to agree excellently with previously reported results for $K=0$ and $S=$

0 . It is found that:

i. For the stretching $\operatorname{cases}(C>0)$, the solution is unique while the dual solutions exist for the shrinking cases $(C<0)$.

ii. The skin friction coefficient and local Nusselt number increases with an increase in suction parameter.

iii. The skin friction coefficient and local Nusselt number decreases with an increase in injection parameter.

iv. As the mass transfer parameter $S$ increases, the range for which the solutions exist is also increases. Therefore, the effect of suction is to widen the range of solutions to exist.

\section{Acknowledgement}

The authors are indebted to the reviewers for their valuable comments and suggestions which led to the improvement of this paper. This research received the financial support from the Ministry of Higher Education (MOHE) under the Fundamental Research Grant Scheme (FRGS/01-01-161841FR5524948).

\section{References}

[1] Wang, C. Y. "Stagnation flow towards a shrinking sheet." International Journal of Non-Linear Mechanics 43, no. 5 (2008): 377-382.

https://doi.org/10.1016/i.ijnonlinmec.2007.12.021

[2] Miklavčič, M., and C. Wang. "Viscous flow due to a shrinking sheet." Quarterly of Applied Mathematics 64, no. 2 (2006): 283-290.

https://doi.org/10.1090/S0033-569X-06-01002-5

[3] Wong, Sin Wei, Abu Omar Awang, and Anuar Ishak. "Stagnation-point flow over an exponentially shrinking/stretching sheet." Zeitschrift für Naturforschung A 66, no. 12 (2011): 705-711.

https://doi.org/10.5560/zna.2011-0037

[4] Nadeem, S., and M. Awais. "Thin film flow of an unsteady shrinking sheet through porous medium with variable viscosity." Physics Letters A 372, no. 30 (2008): 4965-4972.

https://doi.org/10.1016/i.physleta.2008.05.048

[5] Ishak, Anuar, Yian Yian Lok, and loan Pop. "Stagnation-point flow over a shrinking sheet in a micropolar fluid." Chemical Engineering Communications 197, no. 11 (2010): 1417-1427.

https://doi.org/10.1080/00986441003626169

[6] Bhattacharyya, Krishnendu. "Boundary layer flow and heat transfer over an exponentially shrinking sheet." Chinese Physics Letters 28, no. 7 (2011): 074701.

https://doi.org/10.1088/0256-307X/28/7/074701

[7] Bhattacharyya, Krishnendu, and Kuppalapalle Vajravelu. "Stagnation-point flow and heat transfer over an exponentially shrinking sheet." Communications in Nonlinear Science and Numerical Simulation 17, no. 7 (2012): 2728-2734.

https://doi.org/10.1016/i.cnsns.2011.11.011

[8] Bachok, Norfifah, Anuar Ishak, and loan Pop. "Boundary layer stagnation-point flow and heat transfer over an exponentially stretching/shrinking sheet in a nanofluid." International Journal of Heat and Mass Transfer 55, no. 25-26 (2012): 8122-8128.

https://doi.org/10.1016/i.ijheatmasstransfer.2012.08.051

[9] Rohni, Azizah Mohd, Syakila Ahmad, Ahmad Izani Md Ismail, and Ioan Pop. "Boundary layer flow and heat transfer over an exponentially shrinking vertical sheet with suction." International Journal of Thermal Sciences 64 (2013): 264-272.

https://doi.org/10.1016/i.ijthermalsci.2012.08.016

[10] Kasmuri, Juliana, Norfifah Bachok, and Anuar Ishak. "Boundary layer stagnation-point flow and heat transfer past a permeable exponentially shrinking sheet." In AIP Conference Proceedings, vol. 1557, no. 1, pp. 345-349. American Institute of Physics, 2013.

https://doi.org/10.1063/1.4823933 
[11] Rosali, Haliza, Anuar Ishak, Roslinda Nazar, and loan Pop. "Rotating flow over an exponentially shrinking sheet with suction." Journal of Molecular Liquids 211 (2015): 965-969.

https://doi.org/10.1016/j.molliq.2015.08.026

[12] Merkin, J. H., N. Najib, N. Bachok, A. Ishak, and I. Pop. "Stagnation-point flow and heat transfer over an exponentially stretching/shrinking cylinder." Journal of the Taiwan Institute of Chemical Engineers 74 (2017): 65 72. https://doi.org/10.1016/i.jtice.2017.02.008

[13] Kamal, Fatinnabila, Khairy Zaimi, Anuar Ishak, and loan Pop. "Stability analysis on the stagnation-point flow and heat transfer over a permeable stretching/shrinking sheet with heat source effect." International Journal of Numerical Methods for Heat \& Fluid Flow 28 (2018): 2650-2663.

https://doi.org/10.1108/HFF-01-2018-0031

[14] Rahman, A. N. H., N. Bachok, and H. Rosali. "Numerical solutions of MHD stagnation-point flow over an exponentially stretching/shrinking sheet in a nanofluid." In Journal of Physics: Conference Series, vol. 1366, no. 1, p. 012012. IOP Publishing, 2019.

https://doi.org/10.1088/1742-6596/1366/1/012012

[15] Lund, Liaquat Ali, Zurni Omar, and Ilyas Khan. "Steady incompressible magnetohydrodynamics Casson boundary layer flow past a permeable vertical and exponentially shrinking sheet: A stability analysis." Heat Transfer-Asian Research 48, no. 8 (2019): 3538-3556.

https://doi.org/10.1002/htj.21554

[16] Lund, Liaquat Ali, Zurni Omar, and Ilyas Khan. "Quadruple solutions of mixed convection flow of magnetohydrodynamic nanofluid over exponentially vertical shrinking and stretching surfaces: Stability analysis." Computer methods and programs in biomedicine 182 (2019): 105044.

https://doi.org/10.1016/i.cmpb.2019.105044

[17] Ali Lund, Liaquat, Zurni Omar, Ilyas Khan, Jawad Raza, Mohsen Bakouri, and Iskander Tlili. "Stability analysis of Darcy-Forchheimer flow of Casson type nanofluid over an exponential sheet: Investigation of critical points." Symmetry 11, no. 3 (2019): 412. https://doi.org/10.3390/sym11030412

[18] Wahid, N. S., N. M. Arifin, M. Turkyilmazoglu, N. A. A. Rahmin, and M. E. H. Hafidzuddin. "Effect of magnetohydrodynamic Casson fluid flow and heat transfer past a stretching surface in porous medium with slip condition." In Journal of Physics: Conference Series, vol. 1366, no. 1, p. 012028. IOP Publishing, 2019. https://doi.org/10.1088/1742-6596/1366/1/012028

[19] Noormaizatulnazuha, Mohamad, Kamal Fatinnabila, and Zaimi Khairy. "Effect of suction on the stagnation point flow and heat transfer over a stretching/shrinking sheet in a porous medium with stability analysis." Applied Mathematics and Computational Intelligence 8, no. 1 (2019): 17-30.

[20] Wahid, Nur Syahirah, Mohd Ezad Hafidz Hafidzuddin, Norihan Md Arifin, Mustafa Turkyilmazoglu, and Nor Aliza Abd Rahmin. "Magnetohydrodynamic (MHD) Slip Darcy Flow of Viscoelastic Fluid Over A Stretching Sheet and Heat Transfer with Thermal Radiation and Viscous Dissipation." CFD Letters 12, no. 1 (2020): 1-12.

[21] Lund, Liaquat Ali, Zurni Omar, llyas Khan, Jawad Raza, El-Sayed M. Sherif, and Asiful H. Seikh. "Magnetohydrodynamic (MHD) flow of micropolar fluid with effects of viscous dissipation and joule heating over an exponential shrinking sheet: Triple solutions and stability analysis." Symmetry 12, no. 1 (2020): 142. https://doi.org/10.3390/sym12010142

[22] Stewartson, Keith. The theory of laminar boundary layers in compressible fluids. Oxford: Clarendon Press, 1964. https://doi.org/10.1063/1.3051661 